\title{
EXPERIMENT BASED STRUCTURAL STIFFNESS Calibration of a Virtual Robot Model
}

\author{
Gotlih, J.; KARNER, T.; GOTLIH, K. \& BREZOCNIK, M.
}

Abstract: The main advantage of industrial robots is their flexibility, if compared to CNC machines, and speed and accuracy, if compared to humans. To improve accuracy of industrial robots, their stiffness, as one of their most important structural properties needs to be determined. As stiffness of robots with rotary joints is pose dependent, it is convenient to perform FEM simulations to predict a robot's structural stiffness in a desired pose. However, simulation results may deviate in magnitude and trend if compared to measurements. The reasons for deviations are inexact model geometry, friction, component wear and other unknown effects. Our study focuses on structural stiffness measurements for FEM model calibration. A FEM model, with all unknown effects integrated in rotational stiffness' of the robot's joints is created and for each joint, rotational stiffness as a function of joint angles is determined. The calibrated model is used to generate a surrogate model for the robot's structural stiffness.

Key words: Robotics, Stiffness measurement, FEM model calibration, Surrogate model
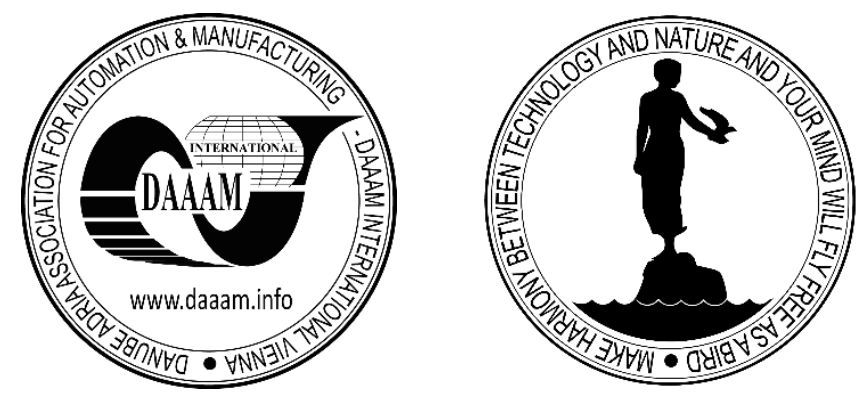

Authors' data: Gotlih, J[anez]*; Assist.Prof. Karner, T[imi]*; Assist.Prof. Assoc.Prof. Gotlih, K[arl]*; Full Prof. Brezocnik, M[iran]*, *Univ. of Maribor, Faculty of Mech. Eng., janez.gotlih@um.si, timi.karner@um.si, karl.gotlih@um.si, miran.brezocnik@um.si

This Publication has to be referred as: Gotlih, J[anez]; Karner, T[imi]; Gotlih, K[arl] \& Brezocnik, M[iran] (2018). Experiment Based Structural Stiffness Calibration of a Virtual Robot Model, Chapter 12 in DAAAM International Scientific Book 2018, pp.131-140, B. Katalinic (Ed.), Published by DAAAM International, ISBN 978-3902734-19-8, ISSN 1726-9687, Vienna, Austria

DOI: $10.2507 /$ daaam.scibook.2018.12 
Gotlih, J.; Karner, T.; Gotlih, K. \& Brezocnik, M.: Experiment Based Structural Sti...

\section{Introduction}

In the era of IoT, demand for unique products is growing. It is increasingly important to produce products as fast, as unique and as accurate as possible. If machine flexibility is increased to produce more unique products, product quality and production speed are sacrificed. Thus, the future calls for more flexible machines then modern machining centres and more accurate than the available robotic mechanisms. Robotic arms show potential to compete with specialized CNC machines, but lack required accuracy to produce parts of high quality, especially when production procedure is associated with high dynamic loads (Höfener \& Schüppstuhl, 2014, Klimchik et al., 2014, Iglesias et al., 2015, Mejri et al., 2015).

For optimization of production system experimental, mathematical and simulation methods are used (Kopac \& Krzic, 2008, Damic et al., 2017, Zhang et al., 2017, Jurkovic et al., 2018). To improve a system its main quality characteristics need to be learned. In case of industrial robots, possibilities to improve their accuracy need to be investigated (Sabourin et al., 2012, Tratar et al., 2014, Denkena et al., 2017). Only after unknown properties become known a robotic system can be calibrated and the planned operations can be optimized (Gotlih et al., 2017, Lin et al., 2017, Lukic et al., 2017, Sun \& Lian, 2018).

Many authors followed this philosophy. A full pose measurement technique of a robots end effector in Cartesian space was performed in (Nguyen et al., 2013). A set of target points was selected and measurements of end effector position and orientation with a laser tracker were performed to avoid the robot's kinematic errors during calibration. After calibration, an increased position and orientation accuracy of the robot was achieved. Proper selection of the robot's poses for calibration experiments was studied in (Wu et al., 2015). Authors proposed a new performance measure and validated the theoretical results with an experimental study. After calibration, a 5.5 times better positioning accuracy was achieved.

Effect of dynamic forces on robot's stiffness was studied in (Cen \& Melkote, 2017). Authors measured the robot's Cartesian stiffness in a series of experiments, using a force scale and a laser displacement sensor. They calculated joint stiffness from the measured Cartesian stiffness with congruence transformation. An similar technique to generate stiffness maps of an industrial robot was used in (Lin et al., 2017). A stiffness performance index was defined, which can be used to analyse the robot's posture influence on its structural stiffness. The problem to design a structure with high stiffness and low mass was recognized in (Sun \& Lian, 2018). Authors managed to increase stiffness of a parallel kinematic machine by three times with less than $20 \%$ increase in mass. Topology of a robot was optimized to maximize its stiffness to mass ratio (Kim et al., 2016). The authors used metamodels to connect the part level mass and stiffness to the robot's structural stiffness.

In our study, we will focus on structural stiffness modelling, which is beside kinematic errors, the most common sources for inaccuracies, as it is present without any dynamic loads acting on the end effector. Our goal is to generate a surrogate model of a robot's structural stiffness in the joint space with regression modelling. 


\section{Materials and methods}

ACMA XR701 robot (figure 1) was chosen for the study, being, due to its load capacity the most suitable robot in our laboratory. First, a digital model of the robot was established. As an original 3D model of the robot was not available it was generated with help of robot manufacturer's manual and data gathered by direct and indirect (3D optical) measurements. A shell model of each component was created and materials were assigned to match the robot's total mass and to achieve a realistic 3D model. The 3D model was verified with the robot's kinematic model, which was created based on the manufacturer's data and tested with the real robot's kinematics. Once the 3D model was validated, a FEM model was created based on the geometry. The FEM model was used for structural stiffness calculations, which resulted in very low deflection values, indicating the necessity to calibrate the model. The results of the first calculation are presented in table 1 .

\begin{tabular}{|c|c|c|c|c|c|}
\hline Pose & $\mathrm{q} 2[\mathrm{deg}]$ & $\mathrm{q} 3$ real $[\mathrm{deg}]$ & $\mathrm{q} 4[\mathrm{deg}]$ & $\mathrm{q} 5[\mathrm{deg}]$ & $\mathrm{Z}$ def. $[\mathrm{mm}]$ \\
\hline 5 & -35 & 40 & 0 & 0 & 0.00022 \\
\hline 41 & 5 & 77.5 & 0 & 0 & 0.0002 \\
\hline 77 & 45 & 115 & 0 & 0 & 0.00027 \\
\hline
\end{tabular}

Tab. 1. Postures and $\mathrm{Z}$ deflection simulation results

Magnitude of deflection in $\mathrm{Z}$ direction (vertical direction) indicated the need to verify the result on measurements. At the same time, an interesting phenomenon was observed. With distance from the robot's main Z-axis (vertical direction through the robot's first axis), deflection is not steadily raising. Instead, minimum deflection was found at pose 41 (table 1), which is located most central in the robot's workspace.

\subsection{Stiffness measurements}

In the second stage, a stiffness measurement experiment was set up. Same load as for simulation was applied to the robot's mount plate at the end effector position to replicate the simulation environment. A digital micrometre was used to measure end effector deflection in $\mathrm{Z}$ direction. The experimental setup is presented on the figure 1 . To model the effect of axis rotation on robot's structural stiffness, an experiment with boundary values presented in table 2 was designed.

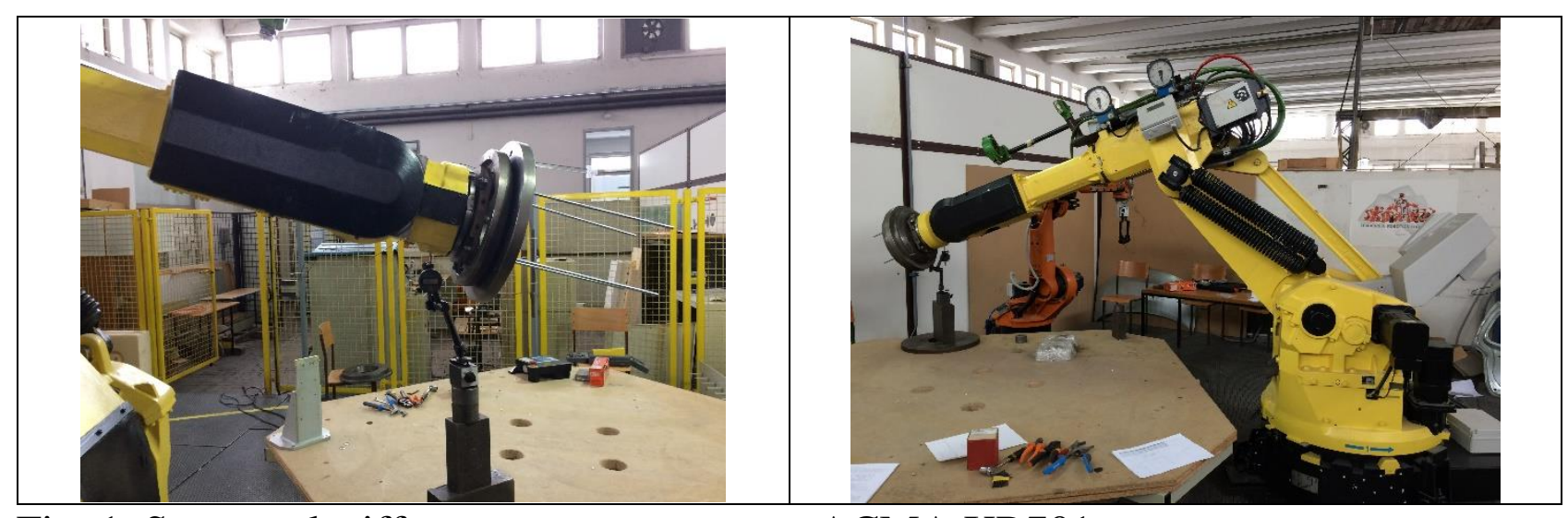

Fig. 1. Structural stiffness measurements on ACMA XR701 
Gotlih, J.; Karner, T.; Gotlih, K. \& Brezocnik, M.: Experiment Based Structural Sti...

\begin{tabular}{|l|l|l|l|}
\hline & Min. [deg] & Mid. [deg] & Max. [deg] \\
\hline q2 & -35 & 5 & 45 \\
\hline q3real & 40 & $77.5(60)$ & $115(77.5)$ \\
\hline q4 & -200 & 0 & 200 \\
\hline q5 & -100 & 0 & 100 \\
\hline
\end{tabular}

Tab. 2. Boundary values for structural stiffness measurement

q2, q3real, q4, q5 are joint 2, 3, 4 and 5 rotations, while minimal and maximal values in table 2 are representing joint rotation boundary values. q3real is used instead of the actual q3 rotation angle, because the robot's structure has a kinematic parallelogram included. Consequently, $\mathrm{q} 3$ does not represent the actual angle between the second and the third link, hence q3real is more convenient to use.

Another consequence of the kinematic parallelogram is kinematic dependency of q3real on $\mathrm{q} 2$, practically meaning that at specific q2 values some q3real values do not exist. To describe the most of the robot's workspace with a manageable number of experiments a full factorial experiment with four factors and three levels had to be adjusted to use the middle and maximum values for q3real, which are the values presented in brackets in table 2 for experiments where $\mathrm{q} 2=-35^{\circ}$. The final matrix consists of 81 experiments. From all experiments, some were inaccessible, therefore nine reference points were selected from the complete matrix. The selected measurement points are presented in table 3 .

Experiments with three different external loads were repeated three or six times. $150 \mathrm{~N}, 375 \mathrm{~N}$ and $650 \mathrm{~N}$ loads were used, where the last load is half of the robot's maximum load capacity. Averaged measurement results are presented in figure 2.

Measured magnitude of deflection in $\mathrm{Z}$ direction is $10^{\wedge} 5$ times higher compared to simulation results in table 1 . Measured deflection results show a similar trend as already observed for simulation results, with a minimum deflection value located at the medium end effector distance from to the robot's main $\mathrm{Z}$ axis. Another interesting observation is that applied load and deflection are not linear. In the following chapters only $650 \mathrm{~N}$ load case are discussed.

\begin{tabular}{|c|c|c|c|c|}
\hline Pose & $\mathrm{q} 2[\mathrm{deg}]$ & $\mathrm{q} 3$ real $[\mathrm{deg}]$ & $\mathrm{q} 4[\mathrm{deg}]$ & $\mathrm{q} 5[\mathrm{deg}]$ \\
\hline 3 & -35 & 40 & -200 & 100 \\
\hline 4 & -35 & 40 & 0 & -100 \\
\hline 5 & -35 & 40 & 0 & 0 \\
\hline 39 & 5 & 77.5 & -200 & 100 \\
\hline 40 & 5 & 77.5 & 0 & -100 \\
\hline 41 & 5 & 77.5 & 0 & 0 \\
\hline 75 & 45 & 115 & -200 & 100 \\
\hline 76 & 45 & 115 & 0 & -100 \\
\hline 77 & 45 & 115 & 0 & 0 \\
\hline
\end{tabular}

Tab. 3. Selected poses for structural stiffness measurements 


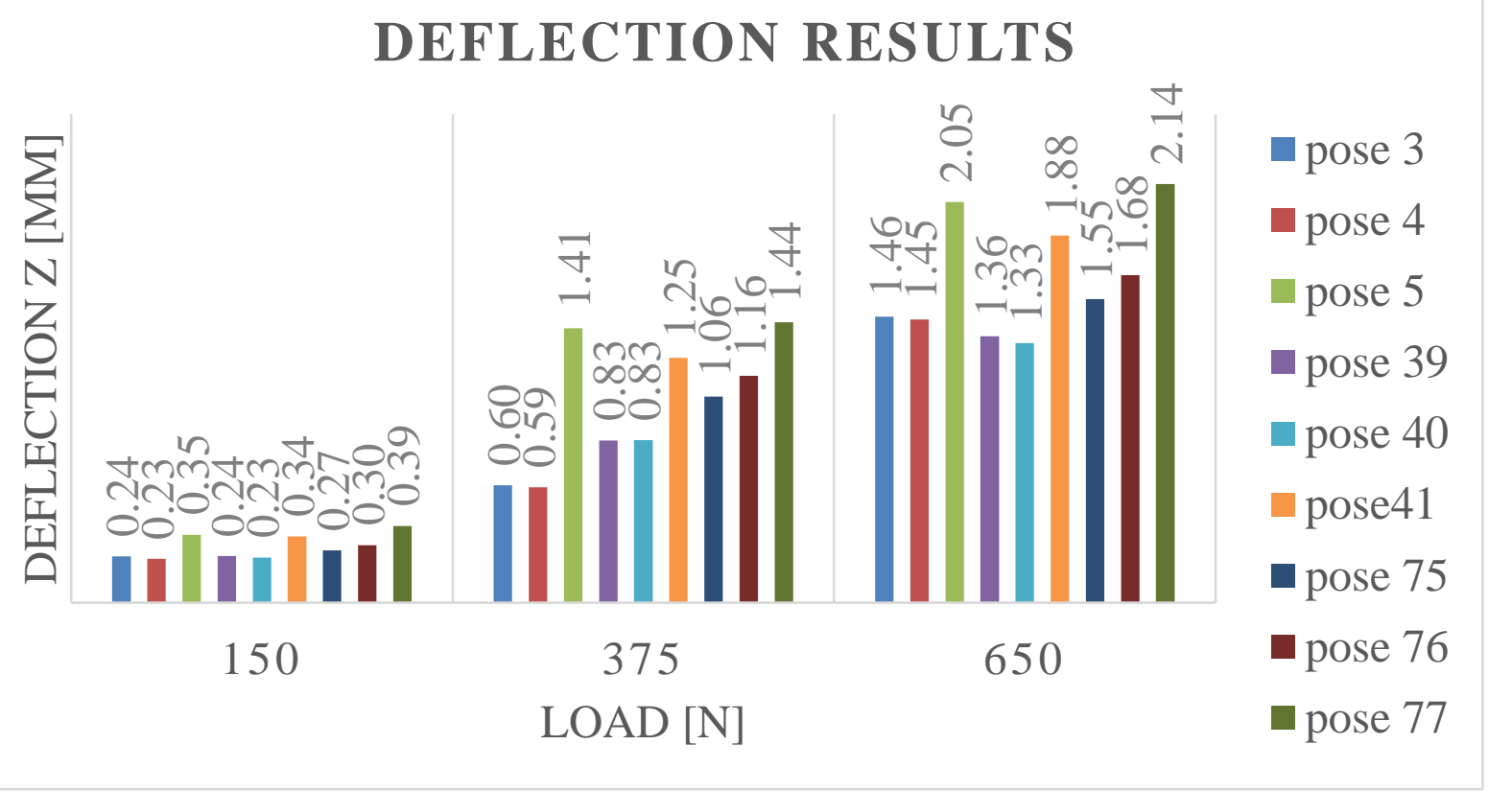

Fig. 2. Deflection measurement results for reference point

\subsection{Virtual model calibration}

Based on measurement results a calibration of the virtual model was performed. A FEM model corresponding to each robot's measurement pose was created and rotational stiffness as a parameter was introduced in each joint as shown in figure 3 . Rotational stiffness as a function of joint angle was introduced to incorporate unknown effects, causing the difference between simulation and measurement results.

To find the dependency between axis rotation and rotational stiffness a two-stage optimization procedure was designed. In the first stage, a sensitivity study was performed to determine the most dominant joint effects. Boundary values for low and high rotational stiffness were set to $10^{\wedge} 5-10^{\wedge} 6 \mathrm{~N} \mathrm{~m} / \mathrm{rad}$.

Results of the study for poses 5, 41 and 77 are presented in figures 4 .

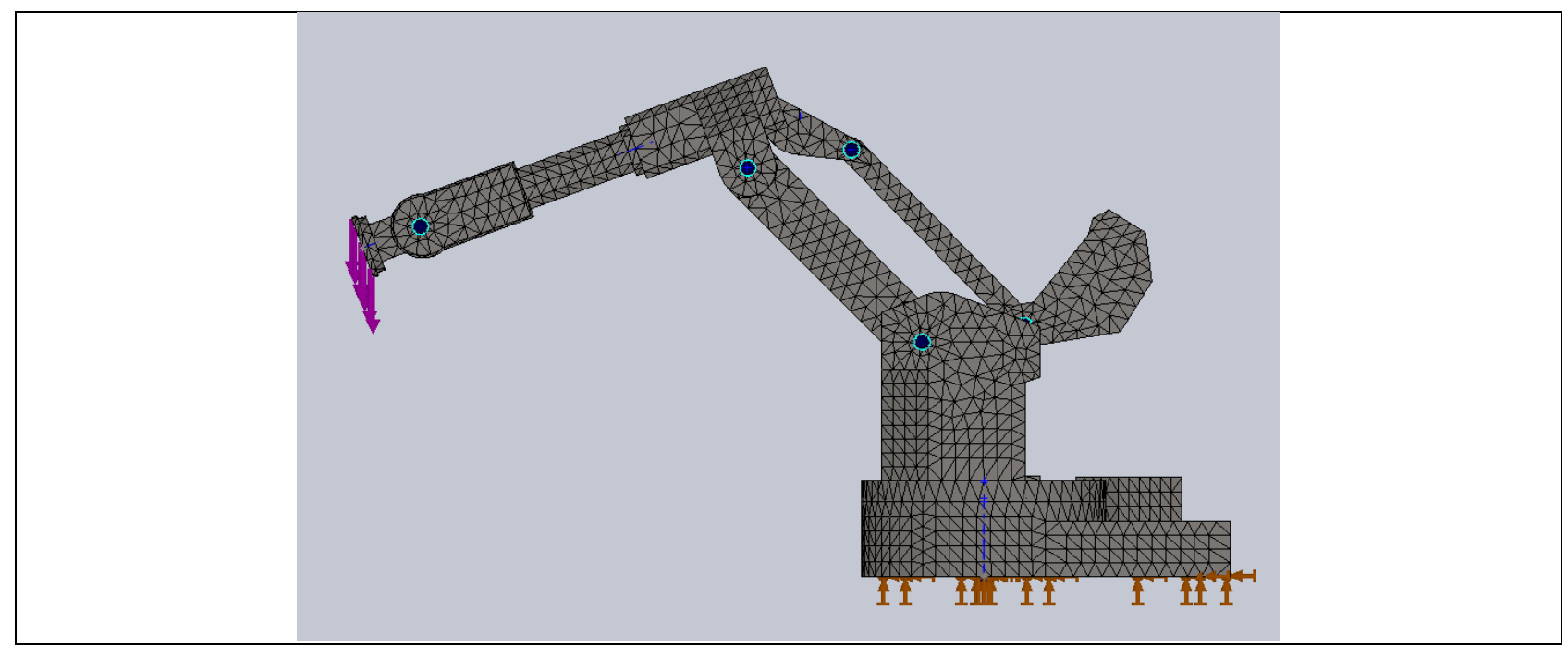

Fig. 3. Virtual model with rotational stiffness parameters and force boundaries 


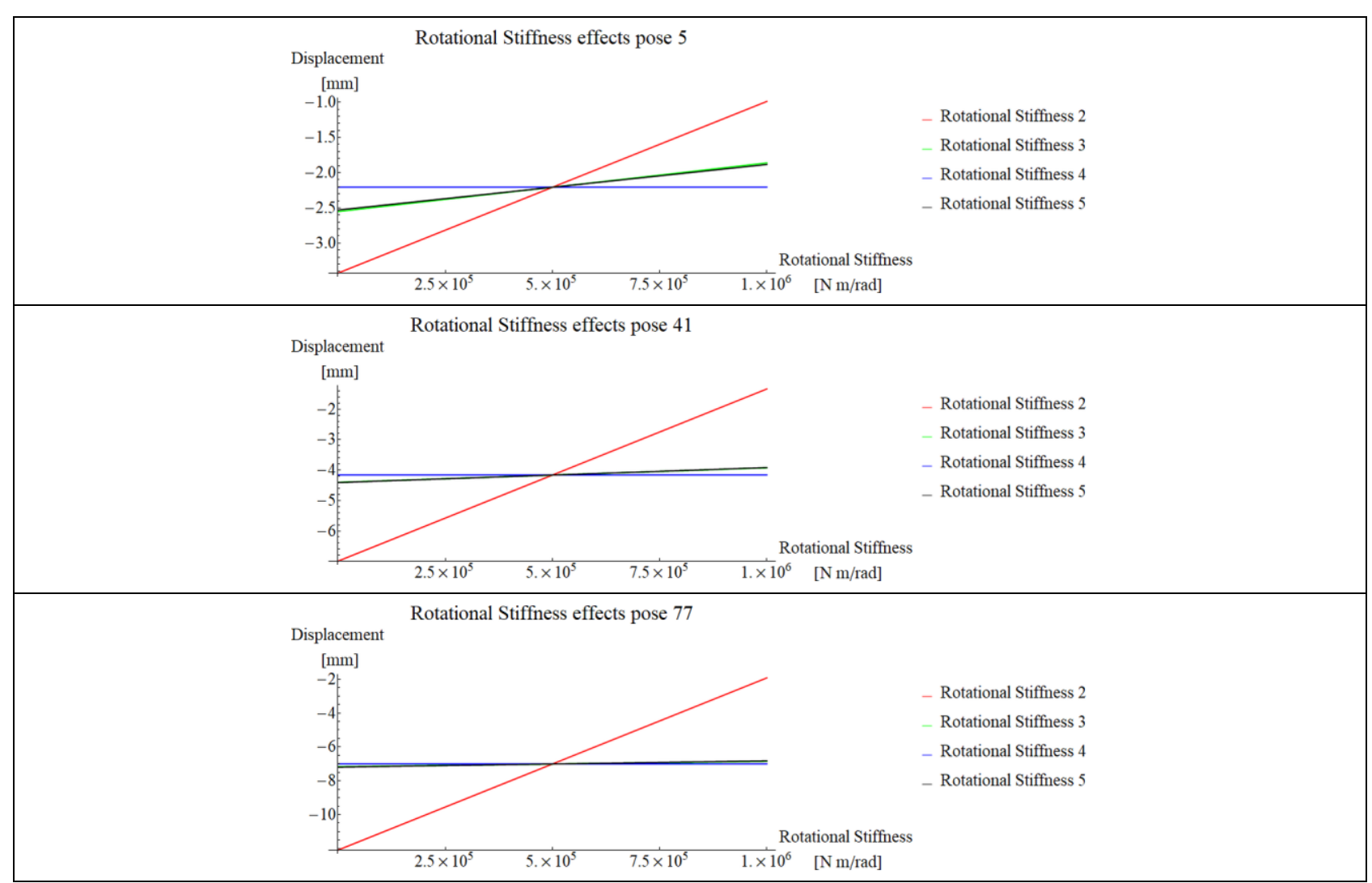

Fig. 4. Rotational stiffness effects on deflection results for poses 5, 41 and 77

Rotational stiffness 2 has a dominant effect on deflection for all poses, followed by rotational stiffness 3 and 5 and rotational stiffness 4 with almost no effect. In the second stage, a three step parametric study was designed to reduce the number of experiments required for calibration. In the first step rotational stiffness 2 and 3 were calibrated on poses 5, 41 and 77 . For the remaining six measured poses, only values for joints 4 and 5 were changed. A two factor, ten level parametric study with the same boundary values as those used for the sensitivity study was created, resulting in 100 experiment runs. This study setup allows adding experiment runs in case of required refinement.

In the second step, poses 4, 40 and 76 were calibrated in a similar way. Rotational stiffness values for joints 2 and 3, determined in the first step were set in the model and only rotational stiffness values for joint 5 were changed. Ten experiments were used. In the third step poses 3, 39 and 75 were calibrated. Rotational stiffness values for joints 2, 3 and 5, determined in the first and second step were set in the model and only rotational stiffness values for joint 4 were changed. Ten experiments were used.

Symmetry points for joints 4 and 5 were added and the rotational stiffness' with the best matching deflection results for each pose were used to fit a regression model connecting rotational stiffness' and joint angles. A sixth degree regression model was chosen to achieve a perfect fit, preserve a natural shape of the response surfaces and reduce gradients in case of small rotations. Results for pose 1 are presented in figures 5. Red points represent optimized rotational stiffness values, while the green planes represent dynamically fitted response surfaces. 


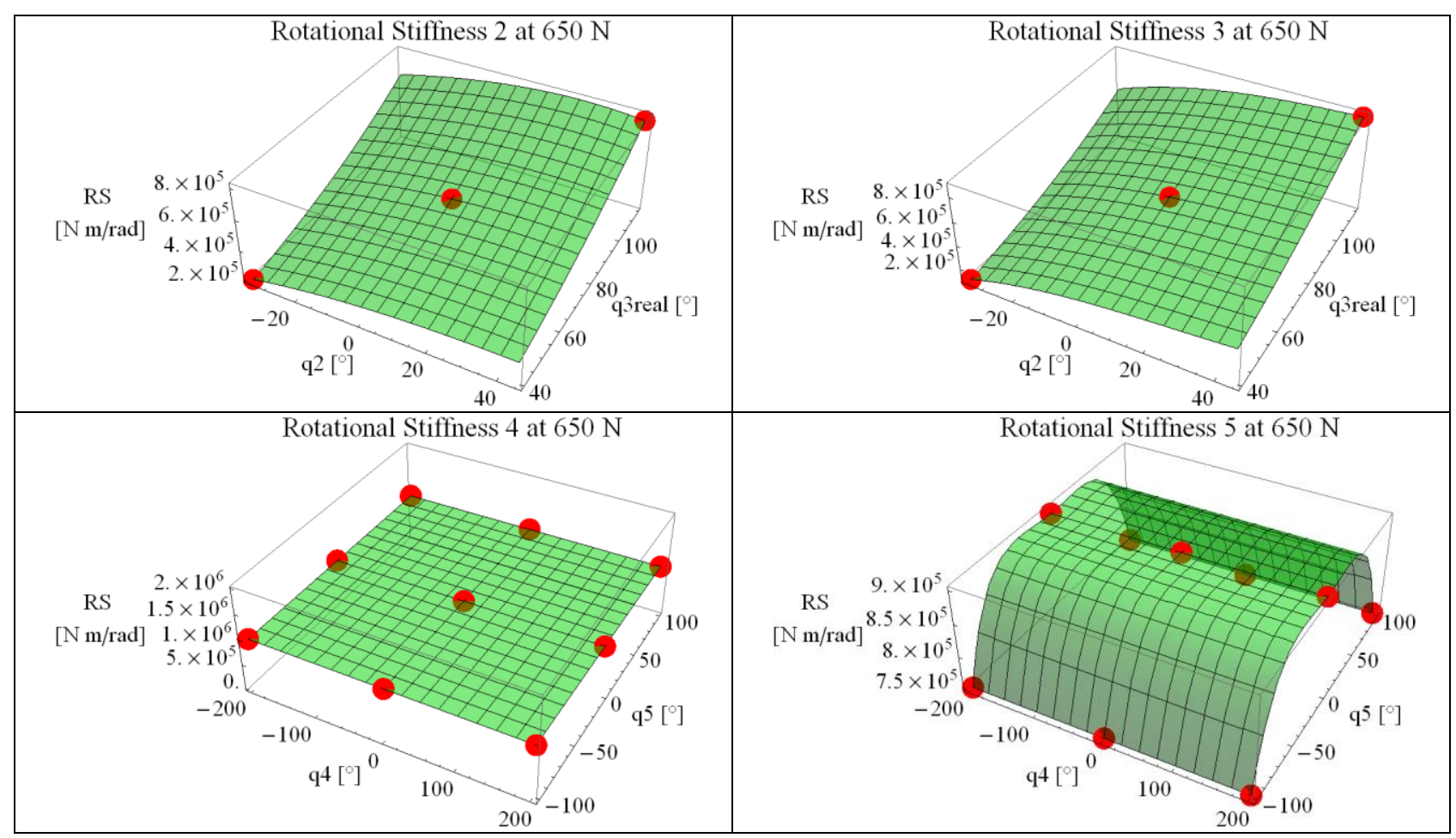

Fig. 5. Response surfaces for pose 1 of rotational stiffness' versus joint angles

\section{Results and discussion}

Finally, the generated rotational stiffness models were integrated into the robot's FEM model and the calibrated model, which was used to calculate the remaining cases of the adjusted full factorial experiment matrix. Main effects of joint angle rotations on deflection results were studied and a structural stiffness surrogate model for the robot was created, by regression modelling.

\subsection{Main effects}

Main effects of joint angle rotation versus normalized structural stiffness are presented in figures 6 . Red curves represent maximum values, green curves represent averaged values and blue curves represent minimum normalized structural stiffness values. q2 effect on normalized structural stiffness shows the expected behaviour. Structural stiffness reduces with distance from the robot's base as showed by the minimum curve, but also the average and the maximum curve are close to the expected behaviour. The adjustment of experiment matrix and consequently lack of experiment orthogonality could be the main reason for the unexpected behaviour. The average curve for q3real shows a good agreement with the expected behaviour. An unexpected drop of maximum structural stiffness is observed at $60^{\circ}$ angle, which is not reflected by the average curve, as minimum curve is raised at that angle. Lack of orthogonality is likely the reason for this behaviour. $\mathrm{q} 4$ has almost no influence on the robot's structural stiffness. q5 shows an interesting behaviour. For some postures, maximum stiffness can be achieved at q $5=0^{\circ}$, but stiffness drops quickly with robot's posture change. This indicates that q5 does not have a dominant effect on the robot's structural stiffness. Stable conditions can be achieved close to q5 boundary values, which is convenient as for $\mathrm{q} 5=0^{\circ}$ the robot is also in its singularity state. 
Gotlih, J.; Karner, T.; Gotlih, K. \& Brezocnik, M.: Experiment Based Structural Sti...

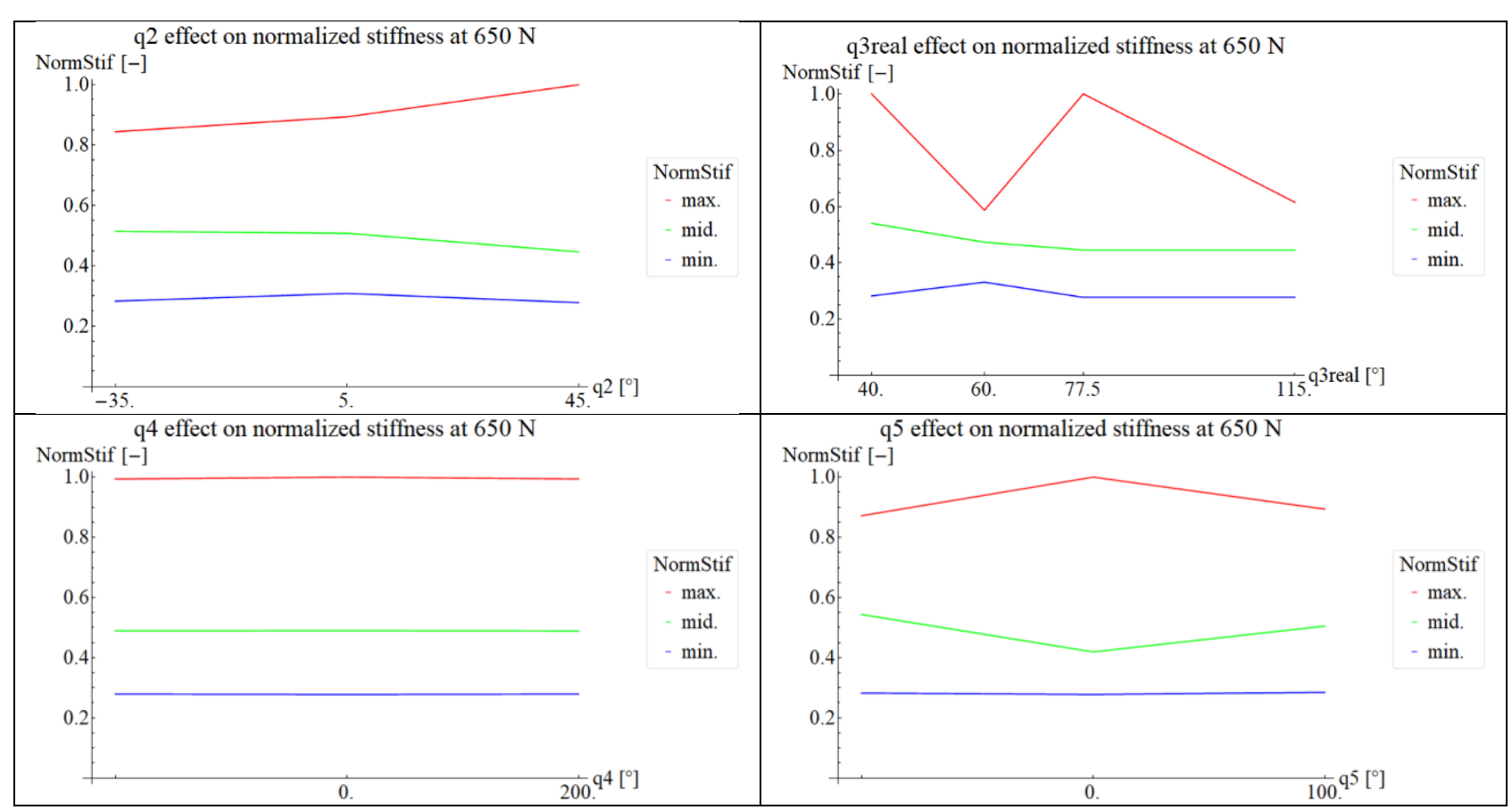

Fig. 6. Main effects of joint (2-5) rotation angle on normalized structural stiffness

\subsection{Surrogate model for robot's structural stiffness}

The robot's kinematic workspace cross section was equipped with its structural stiffness values to generate three dimensional structural stiffness maps. The cross section is three dimensional due to the fourth joint rotation, which moves the end effector point outside the Y zero plane. Warm colours represent high stiffness, while cold colours represent low stiffness. In figures 7, two viewpoints at the same stiffness maps are presented.

The most interesting conclusion regarding figures 7 is that there are regions in the middle of the robot's workspace, where the lowest and the highest structural stiffness are close by. Overall, the results are expected, with low structural stiffness observed at high distance from the robot's main Z-axis and at increased robot's stretch.

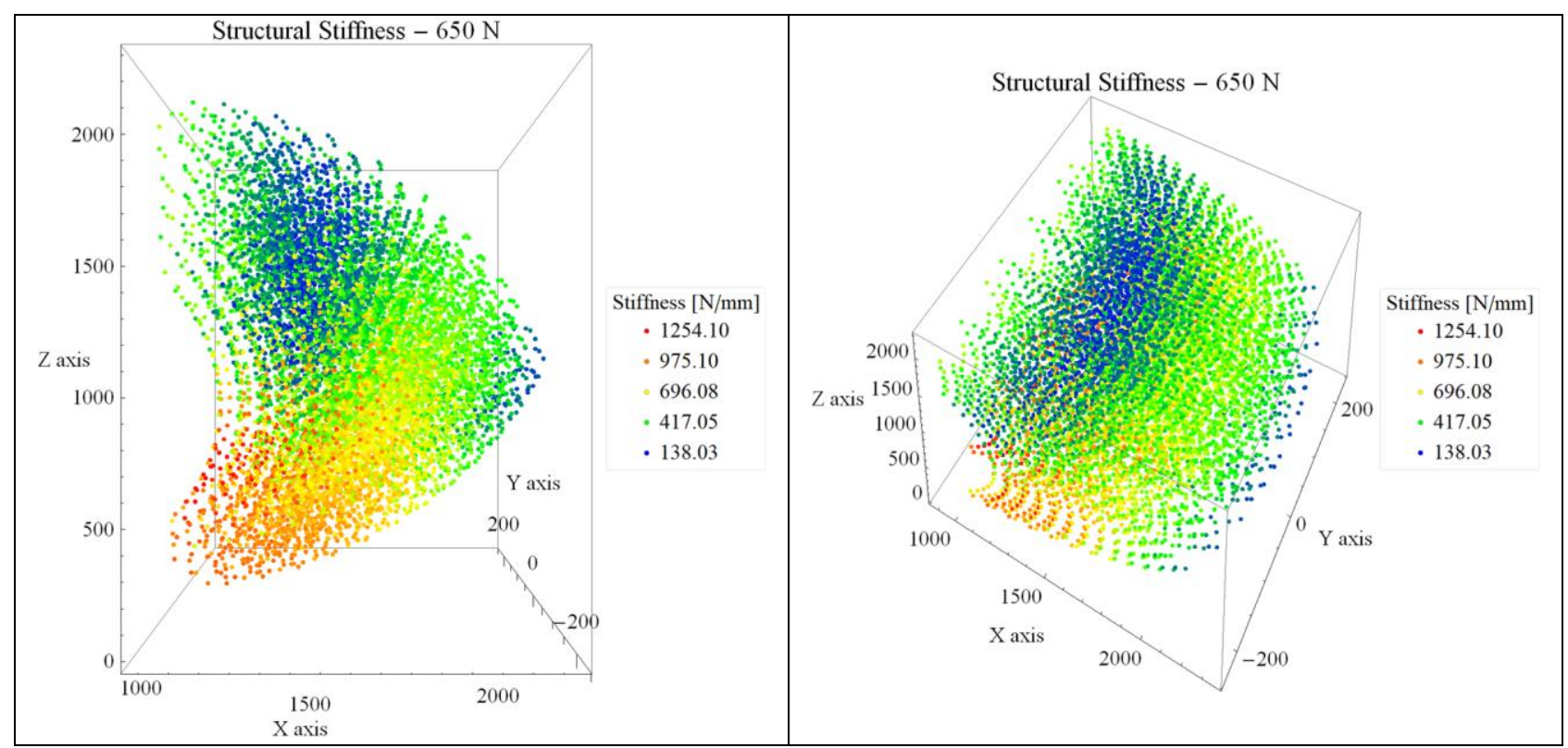

Fig. 7. Structural stiffness map of ACMA XR701 


\section{Conclusion}

The presented paper offers insight into structural properties of an industrial robot. To generate a model for structural stiffness prediction of an industrial robot, a FEM model calibration technique based on structural stiffness measurements and regression modelling was presented. The technique consists of geometrical data acquisition, 3D modelling, 3D model verification, FEM modelling, FEM model verification, stiffness measurements, FEM model calibration and surrogate model creation.

Our conclusion has been that a simple FEM model based on the robot's geometry was not well suited for structural stiffness prediction. Due to geometrical and physical properties of the structure, which could not be accurately included in the model, e.g. friction and wear, the results of the preliminary FEM model were far from the measured results. Experiments to gather relevant data from the actual robot and consecutive parametric studies were performed to calibrate the virtual model. A dedicated set up for robot's stiffness measurements would enable gathering more data to increase calibration quality. Because FEM analyses require skilled engineers for set up and lots of calculation time, a surrogate model for the robot's structural stiffness was created. Main effects were analysed revealing good agreement with the expected behaviour. Observed exceptions were linked to the lack of orthogonality in experiment design, originating from the robot's kinematic structure.

Our conclusion from the structural stiffness maps was that some regions in the robot's workspace are accessible with poses that have far superior structural stiffness to others. With this in mind, an end effector can be mounted to enforce the robot's structural stiffness for a specific task, while the task itself can be optimized on structural stiffness. Our future research will focus on generating kinematic and dynamic performance maps of the robot, to describe the robot's kinematic and dynamic properties.

\section{References}

Cen, L. J. \& Melkote, S. N. (2017). Effect of Robot Dynamics on the Machining Forces in Robotic Milling. 45th Sme North American Manufacturing Research Conference (Namrc 45), Vol 10, (486-496), ISSN: 2351-9789

Damic, V.; Cohodar, M. \& Muratovic, M. (2017). Dynamic Modelling of Mobile Robots Based on its 3D CAD Model. Annals of DAAAM \& Proceedings, Vol 28, (144149), ISSN: 1726-9679

Denkena, B.; Bergmann, B. \& Lepper, T. (2017). Design and optimization of a machining robot. Procedia Manufacturing, Vol 14, (89-96), ISSN: 2351-9789

Gotlih, J.; Brezocnik, M.; Balic, J.; Karner, T.; Razborsek, B. \& Gotlih, K. (2017). Determination of accuracy contour and optimization of workpiece positioning for robot milling. Advances in Production Engineering \& Management, Vol 12, No.3, (233244), ISSN: $1854-6250$ 
Höfener, M. \& Schüppstuhl, T. (2014). A method for increasing the accuracy of "onworkpiece" machining with small industrial robots for composite repair. Production Engineering, Vol 8, No.6, (701-709), ISSN: 1863-7353

Iglesias, I.; Sebastián, M. A. \& Ares, J. E. (2015). Overview of the State of Robotic Machining: Current Situation and Future Potential. Procedia Engineering, Vol 132, (911-917), ISSN: 1877-7058

Jurkovic, M.; Jurkovic, Z.; Buljan, S. \& Obad, M. (2018). An experimental and modelling approach for improving utilization rate of the cold roll forming production line. Advances in Production Engineering \& Management, Vol 13, No.1, (57-68), ISSN: 1854-6250

Kim, B. J.; Yun, D. K.; Lee, S. H. \& Jang, G. W. (2016). Topology optimization of industrial robots for system-level stiffness maximization by using part-level metamodels. Structural and Multidisciplinary Optimization, Vol 54, No.4, (10611071), ISSN: 1615-147x

Klimchik, A.; Wu, Y.; Caro, S.; Furet, B. \& Pashkevich, A. (2014). Accuracy Improvement of Robot-Based Milling Using an Enhanced Manipulator Model, pp. 7381, ISBN: 978-3-319-07058-2, Cham, Springer International Publishing

Kopac, J. \& Krzic, P. (2008). CAM algorithm as important element by achieving of good machined surface quality. Strojniski Vestnik-Journal of Mechanical Engineering, Vol 54, No.4, (280-287), ISSN: 0039-2480

Lin, Y.; Zhao, H. \& Ding, H. (2017). Posture optimization methodology of 6R industrial robots for machining using performance evaluation indexes. Robotics and Computer-Integrated Manufacturing, Vol 48, (59-72), ISSN: 0736-5845

Lukic, D.; Milosevic, M.; Antic, A.; Borojevic, S. \& Ficko, M. (2017). Multi-criteria selection of manufacturing processes in the conceptual process planning. Advances in Production Engineering \& Management, Vol 12, No.2, (151-162), ISSN: 1854-6250 Mejri, S.; Gagnol, V.; Le, T.-P.; Sabourin, L.; Ray, P. \& Paultre, P. (2015). Dynamic characterization of machining robot and stability analysis. The International Journal of Advanced Manufacturing Technology, Vol 82, No.1-4, (351-359), ISSN: 1433-3015 Nguyen, H. N.; Zhou, J. \& Kang, H. J. (2013). A new full pose measurement method for robot calibration. Sensors (Basel), Vol 13, No.7, (9132-9147), ISSN: 1424-8220 Sabourin, L.; Robin, V.; Gogu, G. \& Fauconnier, J. M. (2012). Improving the capability of a redundant robotic cell for cast parts finishing. Industrial Robot-an International Journal, Vol 39, No.4, (381-391), ISSN: 0143-991x

Sun, T. \& Lian, B. B. (2018). Stiffness and mass optimization of parallel kinematic machine. Mechanism and Machine Theory, Vol 120, (73-88), ISSN: 0094-114x

Tratar, J.; Pušavec, F. \& Kopač, J. (2014). Tool wear performance evaluationin MDF machining with anthropomorphic robot. Tehnički vjesnik, Vol 21, No.4, (911-915), ISSN: $1848-6339$

Wu, Y.; Klimchik, A.; Caro, S.; Furet, B. \& Pashkevich, A. (2015). Geometric calibration of industrial robots using enhanced partial pose measurements and design of experiments. Robotics and Computer-Integrated Manufacturing, Vol 35, (151-168), ISSN: 0736-5845

Zhang, H.; Liu, S.; Moraca, S. \& Ojstersek, R. (2017). An Effective Use of Hybrid Metaheuristics Algorithm for Job Shop Scheduling Problem. International Journal of Simulation Modelling, Vol 16, No.4, (644-657), ISSN: 1726-4529 